\title{
An effective mouse model for adoptive cancer immunotherapy targeting neoantigens
}

\author{
Ken-ichi Hanada, ${ }^{1,2}$ Zhiya Yu, ${ }^{1,2}$ Gabrielle R. Chappell, ${ }^{1,2,3}$ Adam S. Park, ${ }^{1,2,4}$ and Nicholas P. Restifo, \\ 'Surgery Branch, National Cancer Institute (NCI), NIH, Bethesda, Maryland, USA. ²Center for Cell-Based Therapy, NCI, NIH, \\ Bethesda, Maryland, USA. ${ }^{3}$ Biomedical Sciences Research Complex, University of St Andrews, North Haugh, St Andrews, \\ United Kingdom. ${ }^{4}$ Harvard University, Cambridge, Massachusetts, USA.
}

\begin{abstract}
The adoptive cell transfer (ACT) of T cells targeting mutated neoantigens can cause objective responses in varieties of metastatic cancers, but the development of new T cell-based treatments relies on accurate animal models. To investigate the therapeutic effect of targeting a neoantigen with ACT, we used T cells from pmel-1 T cell receptor-transgenic mice, known to recognize a WT peptide, gp100, and a mutated version of the peptide that has higher avidity. We gene-engineered B16 cells to express the WT or mutated gp100 epitopes and found that pmel-1-specific T cells targeting a neoantigen tumor target augmented recognition as measured by IFN- $\gamma$ production. Neoantigen expression by B16 also enhanced the capacity of pmel-1 T cells to trigger the complete and durable regression of large, established, vascularized tumor and required less lymphodepleting conditioning. Targeting neoantigen uncovered the possibility of using enforced expression of the IL-2R $\alpha$ chain (CD25) in mutation-reactive CD8+ $T$ cells to improve their antitumor functionality. These data reveal that targeting of "mutated-self" neoantigens may lead to improved efficacy and reduced toxicities of $\mathrm{T}$ cell-based cellular immunotherapies for patients with cancer.
\end{abstract}

\section{Authorship note: $\mathrm{KH}$ and $\mathrm{ZY}$ contributed equally to this work. \\ Conflict of interest: The authors have declared that no conflict of interest exists.}

Copyright: (c) 2019 American Society for Clinical Investigation

Submitted: August 22, 2018

Accepted: April 17, 2019

Published: May 16, 2019.

Reference information: /CI Insight. 2019;4(10):e124405. https://doi. org/10.1172/jici.insight.124405.

\section{Introduction}

$\mathrm{T}$ cells play a central role in antitumor immunity. This is vividly illustrated using adoptive cell transferbased (ACT-based) immunotherapies, which involve the administration of $\mathrm{T}$ cells grown ex vivo. In the solid tumor setting, some ACT approaches have targeted tissue differentiation antigens - normal proteins that are overexpressed in cancers $(1,2)$. Generating self-reactive cells is more difficult because of immunological self-tolerance, a physiological process that eliminates or functionally neutralizes autoreactive $\mathrm{T}$ cells $(3,4)$. If successful, targeting self-antigens can lead to off-tumor, on-target toxicities (5).

It seems plausible that targeting "neoantigens" will enable greater tumor specificity. Tumor-specific peptides are a result of somatic mutation and expression of aberrant proteins. Mutations can be caused by DNA-damaging agents such as UV light, chemical carcinogens, or unrepaired errors during DNA replication $(6,7)$. The reasons for the great susceptibility of melanoma to immune-based therapies are not fully understood but are due in part to the demonstrable fact that melanomas contain large numbers of neoantigens (8).

Improvement of antitumor responses and reactivity in tumor-infiltrating lymphocyte (TIL) therapy may be achievable through targeting of mutated neoantigens. Examples of neoantigen-targeted $\mathrm{T}$ cells with remarkable clinical responses include a metastatic cholangiocarcinoma patient treated with $\mathrm{CD} 4^{+} \mathrm{T}$ cells reactive to a mutation in ERBB2-interacting protein (9), a metastatic colon cancer patient treated with KRAS G12D mutation-reactive $\mathrm{CD}^{+} \mathrm{T}$ cells (10), and a patient with chemorefractory hormone receptorpositive metastatic breast cancer who was treated with both $\mathrm{CD} 4^{+}$and $\mathrm{CD} 8^{+} \mathrm{T}$ cells reactive to multiple neoantigens, including SLC3A2, KIAA0368, CADPS2, and CTSB (11). However, the response rate of mutation-reactive TIL ACT therapy has been low, and further improvement is necessary (12).

Improvement of ACT-based approaches has relied in large part on the use of animal models, but many models currently used fail to accurately mirror human malignancy and lack valuable predictive power. The treatment of very early or small tumors that have not fully established independent vasculature can fail to model key aspects of tumor treatment required in patients bearing tumors that have been established for months or years $(13,14)$. Some models are induced using high doses of chemical carcinogens that may overshoot the induc- 
tion of neoantigens compared with their human counterparts $(13,15,16)$. Conversely, transgenic mice carrying mutated oncogenes and tumor suppressor genes may express very few neoantigens compared with most human malignancies (17). In addition, some of the genetically engineered mouse models have uneven tumor growth rates, which can present practical complications in the design of large preclinical studies (18). Thus, efforts to develop "mutated-self" antigen models might facilitate the development of novel cell-based treatments.

In our previous studies, we showed that pmel-1 CD8 $8^{+} \mathrm{T}$ cells react to the nonmutated epitope (EGSRNQDWL) derived from the melanoma differentiation antigen Pmel, or gp100 $(19,20)$. pmel-1 CD8 ${ }^{+} \mathrm{T}$ cells can destroy subcutaneously established B16 melanoma, albeit inefficiently. We also found that pmel-1 T cells recognize the human gp100-derived (KVPRNQDWL) epitope (19). This presented an opportunity to create a neoantigen model in B16 cells by genetically introducing a chimeric mouse gp100 with human gp100-derived amino acid sequences (KVP) at positions 25-27. The EGS to KVP mutation does not represent a single-base pair point mutation of the kind often studied; however, it might mimic mutations involving insertions or deletions, especially those involving translocations, frameshift mutations, loss of stop codon mutations, or mutations resulting in aberrant splicing. The altered epitope represents a "mutated-self," and hence a model for neoantigen targeting by ACT. We report here that $\mathrm{B} 16_{\mathrm{KVP}}$ may serve as a model that is highly relevant to the development of ACT-based immunotherapies targeting tumor-specific neoantigens in the clinic.

Various effects have focused on augmenting the persistence and function of adoptively transferred $\mathrm{T}$ cells to improve response rates. Our findings suggested that successful ACT treatment of neoantigen-expressing B16 tumors may not require lymphodepletion or recombinant vaccination to achieve tumor regression and clearance. Additionally, using our neoantigen-expressing tumor model, we investigated the effect of $\mathrm{T}$ cell modifications on ACT. We found that insertion of the IL-2R $\alpha$ chain (CD25) into CD8 ${ }^{+} \mathrm{T}$ cells improved their ability to treat established tumors, a finding not observed using the previous "self-antigen" model. We present the characteristics of a new "mutated-self" model and propose its usefulness through presenting an example of a T cell modification that is effective in the neoantigen setting. The unique ability to compare the effect of T cell modifications on ACT therapy in either the autoantigen setting or the neoantigen setting by switching tumors between $\mathrm{B} 16_{\mathrm{EGS}}$ and $\mathrm{B} 16_{\mathrm{KVP}}$ is of significant value in understanding how to best treat patients with these tumors.

\section{Results}

Neoantigen expression improves B16 tumor recognition by pmel-1 T cells. Melanoma has been a source of intensive study in cancer immunology for many years because it often gives rise to endogenous $\mathrm{T}$ cells with antitumor activity (8). The mechanisms underlying melanoma's susceptibility to immune-based therapies are due in part to the demonstrable fact that melanomas express large numbers of neoantigens (8) and tumor-infiltrating lymphocytes (TILs) that are reactive to their unique mutations (21). T cells recognize antigens when they are presented on an MHC molecule. B16, a mouse melanoma line, is commonly used to model human melanoma and cancer immunotherapy. B16 is poorly immunogenic and expresses low levels of MHC-I. Furthermore, the mouse autoantigen gp $100_{\mathrm{EGS}}$ has a low affinity to the class I MHC restriction element $\mathrm{H}-2 \mathrm{D}^{\mathrm{b}}$.

We sought to examine whether B16 tumors expressing a neoantigen were more immunogenic and better recognized by pmel-1 T cells. We previously found that when the autoantigen gp $100_{\mathrm{EGS}}$ and neoantigen gp $100_{\mathrm{KVP}}$ peptides were exogenously pulsed onto EL-4 cells, pmel-1 cells recognized gp $100_{\mathrm{KVP}}$ a thousandfold better than the gp $100_{\mathrm{EGS}}$ epitope (19). We thus constructed a control vector encoding the full-length WT mouse gp100 and a vector encoding the full-length mouse gp100 but with amino acids at positions 25-27 changed from EGS to KVP (Figure 1A).

We found that expression of gp $100_{\mathrm{EGS}}$ or $\mathrm{gp} 100_{\mathrm{KVP}}$ was similar among the derivative tumors (Figure $1 \mathrm{~B}$ ). We observed that parental B16 tumor cells upregulated the expression of $\mathrm{H}-2 \mathrm{D}^{\mathrm{b}}$ dramatically in response to IFN- $\gamma$, but the constitutive expression of $\mathrm{H}-2 \mathrm{D}^{\mathrm{b}}$ remained low in comparison with other murine tumor lines such as colorectal adenocarcinoma MC38 and methylcholanthrene-induced fibrosarcoma MCA205 (Figure $1 \mathrm{C}$ ). We therefore made a retrovirus vector encoding $\mathrm{H}-2 \mathrm{D}^{\mathrm{b}}$ to examine whether increased constitutive class I MHC expression resulted in greater tumor recognition by pmel-1 T cells (Figure 1A).

To assess the ability of pmel-1 T cells to recognize candidate B16 tumor models, we measured IFN- $\gamma$ production in an ex vivo coculture assay. We found that recognition of the parental B 16 or B16 $6_{\mathrm{EGS}}$ tumor by pmel-1 T cells was highly dependent on increased expression of the restricting histocompatibility antigen $\mathrm{H}-2 \mathrm{D}^{\mathrm{b}}$ (Figure 1D). In the absence of enforced $\mathrm{H}-2 \mathrm{D}^{\mathrm{b}}$ expression, there was minimal IFN- $\gamma$ production in the coculture. In stark contrast to these tumors, $\mathrm{B} 16_{\mathrm{KVP}}$ without the $H 2-D 1$ transduction was well rec- 
ognized by pmel-1 cells (Figure 1D). This could be explained by enhanced affinity of the KVP epitope to $\mathrm{H}-2 \mathrm{D}^{\mathrm{b}}$ molecules. Not surprisingly, pmel-1 T cells produced significantly more IFN- $\gamma$ when cocultured with $\mathrm{B} 16_{\mathrm{KVP}} / \mathrm{D}^{\mathrm{b}}$ tumor than with B16 $6_{\mathrm{KVP}}$ tumor. Accordingly, we successfully established a panel of B16 derivatives including a neoepitope model with differential ex vivo recognition by pmel-1 T cells.

Targeting neoantigen with ACT increases B16 tumor regression. We sought to elucidate whether enhanced $\mathrm{T}$ cell recognition in our model using the gp $100_{\mathrm{KVP}}$ neoantigen translated to increased tumor regression in vivo. Having observed significant recognition of $\mathrm{B} 16_{\mathrm{KVP}}$ tumors by pmel-1 cells, we examined the efficacy of neoantigen-targeted ACT therapy involving lymphodepletion (22), recombinant vaccination, and IL-2 administration to treat tumor-bearing mice (Figure 2A).

Without treatment, B16 and all 5 of its derivative lines had similarly robust tumor growth rate in C57BL/6 mice (Figure 2B). When $1 \times 10^{6}$ pmel-1 cells were transferred with lymphodepletion and IL-2 (but without vaccination), treatment had little impact on the parental B16 tumors, but it impeded the growth of $\mathrm{B} 16_{\mathrm{EGS}}$ tumors. The $\mathrm{B} 16_{\mathrm{KVP}}$ as well as $\mathrm{B} 16_{\mathrm{EGS}} / \mathrm{D}^{\mathrm{b}}$ tumors transiently regressed during the first 2 weeks after the ACT. However, we found that the same therapy had significantly better efficacy when targeting $\mathrm{B} 16_{\mathrm{KVP}} / \mathrm{D}^{\mathrm{b}}$ tumors $(P<0.05)$. Addition of recombinant vaccination to the ACT regimen resulted in a significant tumor response in all tumors $(P<0.05)$. This result was particularly evident in mice with B16 tumors presenting the neoantigen. In B16 tumors expressing the mutated epitope $\left(\mathrm{gp} 100_{\mathrm{KVP}}\right), 4$ of 5 mice were tumor-free 55 days after cell transfer with the tripartite regimen (Figure 2B).

Because we observed significant regression of $\mathrm{B} 16_{\mathrm{KVP}} / \mathrm{D}^{\mathrm{b}}$ tumors in response to ACT treatment without vaccination, we sought to investigate whether this effect was caused by direct pmel-1 $\mathrm{T}$ cell recognition or by "self" vaccinations through cross-presentation by host antigen-presenting cells (APCs). We treated B16 ${ }_{\mathrm{KVP}} / \mathrm{D}^{\mathrm{b}}$ tumors with pmel-1 ACT in the WT and in $\beta_{2}$-microglobulin-knockout mice that do not have functional class I MHC molecules to present antigens to pmel-1 $\mathrm{T}$ cells. We found that although a boost of tumor treatment efficacy by vaccination with recombinant vaccinia virus encoding human gp100 was host-APC-dependent, in the absence of vaccination, tumor treatment effects by pmel-1 ACT remained the same in both the WT and the $\beta_{2}$-microglobulin-knockout mice (Figure 2C). Therefore, we concluded that the therapeutic effect of pmel-1 cells in the absence of vaccine was through direct recognition of neoantigen presented on the tumor cells. This finding further prompted us to investigate whether pmel-1 transfer and vaccination were sufficiently effective to cause regression of neoantigen-expressing tumors without lymphodepletion or IL-2 administration.

Neoantigen expression enables successful tumor treatment without lymphodepletion. In our previously established model, tumor-reactive pmel-1 T cells with irradiation or IL-2 alone were not enough to trigger regression of autoantigen-expressing B16 tumors. The best response in our autoantigen model involved the classical tripartite regimen including irradiation, recombinant vaccination, and IL-2 (19). Observing the immunogenicity of gp $100_{\mathrm{KVP}}$ and therapeutic significance of recombinant vaccination in the "mutated-self" model, we investigated whether all elements of the tripartite ACT regime were necessary in neoantigen-targeted therapies.

Using B16 $6_{\mathrm{KVP}} / \mathrm{D}^{\mathrm{b}}$-expressing tumors, we investigated the contribution of each component of the current ACT tripartite regimen with titrated numbers of pmel-1 cells (Figure 3A). Without vaccination or total-body irradiation of the hosts, the dose of pmel-1 cells needed to achieve a statistically significant treatment was $2 \times 10^{7} \mathrm{cells}$, which was 100 times greater than the dose required to produce similar treatment effect in irradiated hosts. Even at the $2 \times 10^{7}$ dose, without irradiation, the tumor regression was transient, and all mice relapsed with none experiencing complete regression (Figure 3B). With irradiation but without vaccination is a condition that is like our current clinical ACT protocols. Under this condition, we observed significant regression of the tumor for approximately 20 days $(P<0.05)$ (Figure 3A), and 1 of 10 mice that received $2 \times 10^{7}$ pmel- 1 cells was tumor-free 60 days after ACT (Figure 3B). We thus observed enhanced responses at the higher numbers of the cells, again showing the importance of giving the maximum number of $\mathrm{T}$ cells possible under this condition.

Vaccination significantly enhanced antitumor effects of pmel-1 $\mathrm{T}$ cells regardless of irradiation status of the hosts even at the lowest cell dose of $2 \times 10^{5}$ cells (Figure 3A). As few as $2 \times 10^{5}$ pmel- 1 cells resulted in the complete regression of 4 of 10 mice, which remained tumor-free for more than 60 days after the ACT. At the maximum cell dose, 9 of 10 irradiated mice and 4 of 10 nonirradiated mice were tumor-free 60 days after cell transfer and vaccination (Figure $3 \mathrm{~B}$ ). Therefore, combination of a large number of cells and vaccination could achieve tumor clearance without lymphodepleting irradiation.

We sought to test the contribution of vaccination and irradiation in ACT treatment of neoantigen-expressing tumor with low MHC-I expression, B16 ${ }_{\mathrm{KVP}}$ (Figure 4A). We observed similar results compared with those 
A

(i) MSGV1 gp100EGS V5 IRES BSR

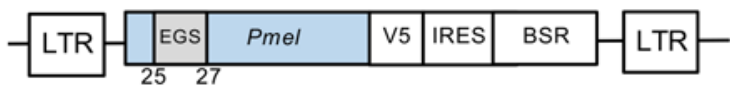

(ii) MSGV1 gp100 kvP V5 IRES BSR

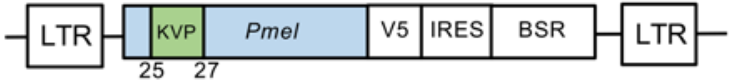

(iii) MSGV1 H-2D ${ }^{\mathrm{b}}$ IRES PURO

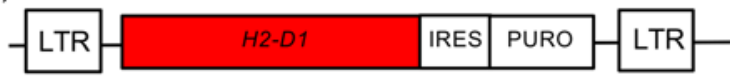

C

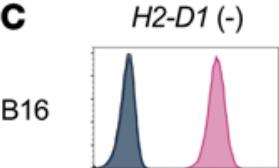

B16
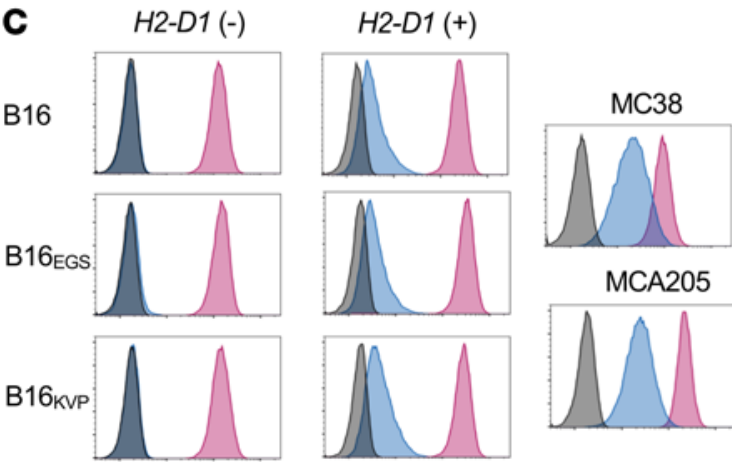

MCA205
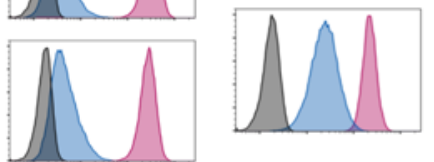

$\mathrm{H}-2 \mathrm{D}^{\mathrm{b}} \mathrm{FITC}$

$\square$ Isotype

$\square \operatorname{IFN} \gamma(-)$

$\operatorname{IFN} \gamma(+)$
B
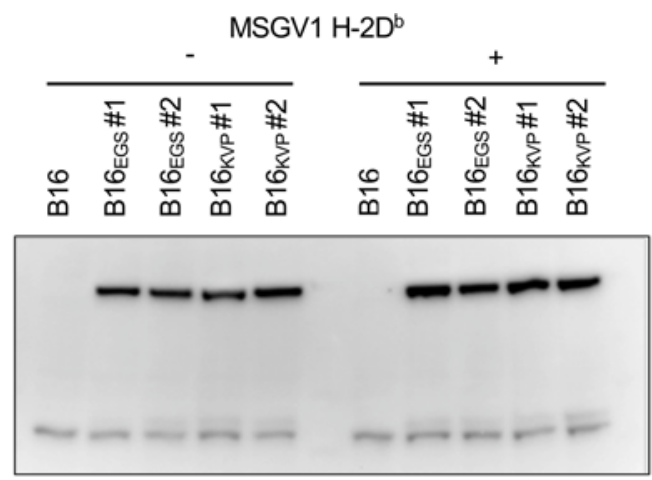

gp100:

$66 \mathrm{kD}$

$\beta$-actin: $41 \mathrm{kD}$

Figure 1. Modified B16 cell lines and their recognition by pmel-1 T cells. (A) Schematic diagram. Retroviral vectors for genetic modifications: MSCV1 vectors containing cDNAs of (i) native mouse gp100, (ii) mouse gp100 modified at amino acid positions 25-27, or (iii) H-2 $D^{\mathrm{b}}$ were constructed. LTR, long-terminal repeat; EGS, Glu-Gly-Ser; KVP, Lys-Val-Pro; V5, V5 tag; IRES, internal ribosome entry site; BSR, blasticidin S resistance gene; PURO, puromycin resistance gene. (B) Western blot of cell lysates from modified B16 tumors probed by an anti-V 5 tag antibody and an anti- $\beta$-actin antibody (loading control); \#1 and \#2 represent 2 retroviral plasmid preparations used for the transduction. (C) $\mathrm{H}-2 \mathrm{D}^{\mathrm{b}}$ expression analysis on modified B16, MC38, and MCA205 cells by flow cytometry. (D) ELISA measuring tumor recognition by pmel-1 T cells in coculture assay: $1 \times 10^{5}$ pmel-1 T cells were cocultured with the same number of tumor cells for 24 hours. IFN- $\gamma$ concentration in the culture supernatant was measured by ELISA. The results represent 1 of 3 independent experiments. Error bars indicate the mean $\pm S D ; n=3$. ${ }^{*} P<0.01$ by ANOVA followed by Tukey's multiple-comparisons test.

from treatment of $\mathrm{B} 16_{\mathrm{KVP}} / \mathrm{D}^{\mathrm{b}}$ tumor. Therefore, irrespective of the constitutive MHC expression, the tripartite ACT regime was the most effective therapy for targeting neoantigen-expressing tumors. However, we found that significant tumor regression could be achieved with either lymphodepletion or recombinant vaccination.

In our current clinical ACT protocols, patients are treated with lymphodepletion and IL-2 without vaccination. IL-2 is a well-known T cell growth factor and was necessary for the pmel-1 ACT therapy to work against the parental B16 cells (19). However, administration of IL-2 is often associated with severe side effects such as renal toxicities and vascular leak syndrome in human subjects. Accordingly, we reexamined the necessity of IL-2 in this neoantigen mode. Without lymphodepleting irradiation, no tumor regression was observed (Figure 4B). When lymphodepletion was added to the regimen, pmel-1 cells had similar therapeutic effect on tumor growth with or without exogenously provided IL-2, in this experimental setting (Figure 4B).

CD25 expression by pmel-1 cells improves neoantigen-targeted adoptive T cell transfer therapy. IL-2 may be an essential part of current ACT TIL therapy; however, considering the risks of renal toxicity and vascular leak syndrome, lowering the dosage of IL-2 given to patients could decrease biological stress and toxicity, making our therapies more tolerable. A previous study by Su et al. showed that a complex of IL-2 and its antibody could stabilize IL-2 receptor on T cell surface and sustain IL-2 signaling (23). We therefore investigated whether increasing the IL-2 receptor $\alpha$ subunit (CD25) expression on the surface of pmel-1 cells could enhance antitumor activity. We tested this by constitutively expressing high amounts of CD25 (IL-2R $\alpha$ ) and examining the resulting effect in the autoantigen and neoantigen settings. 
A

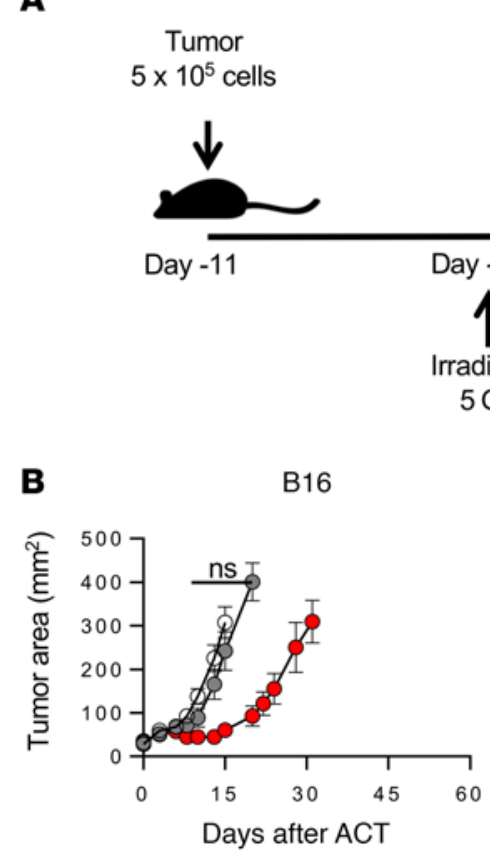

$B 16 / D^{b}$

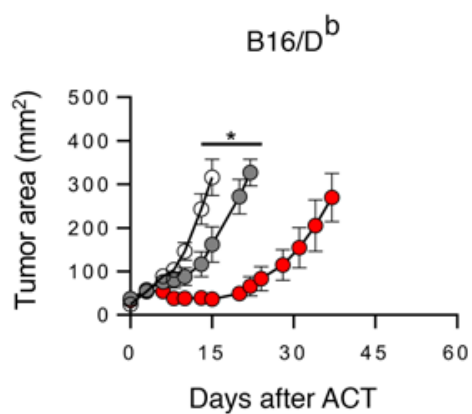

Days after ACT

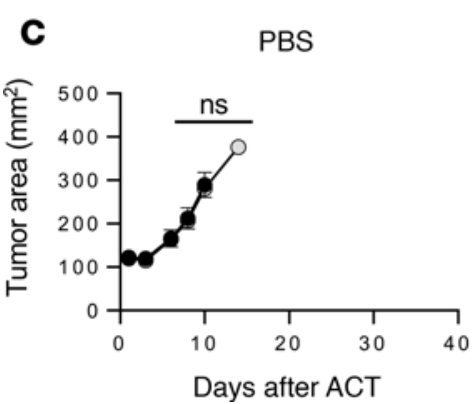

Pmel-1

$2 \times 10^{5}-2 \times 10^{7}$ cells

+ - rVWhgp 100

$2 \times 10^{7} \mathrm{pfu}$

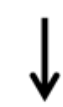

Monitor tumor size

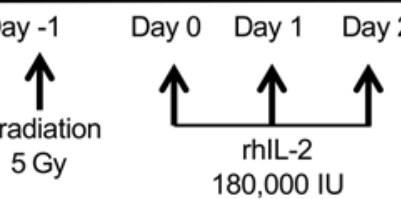

180,000 IU

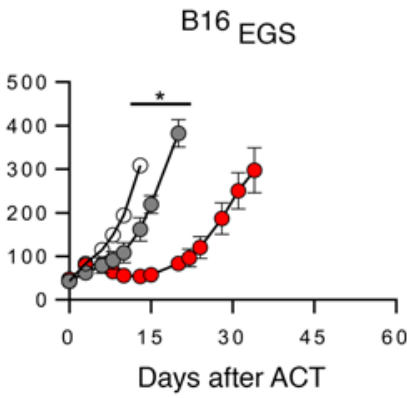

${ }^{B 16}$ EGS $/ D^{b}$

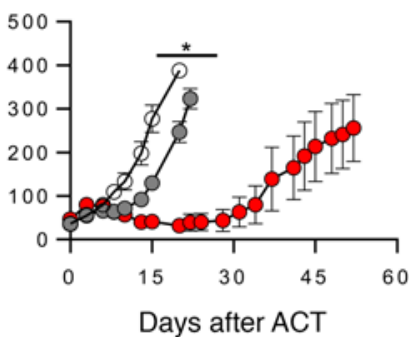

Days after ACT

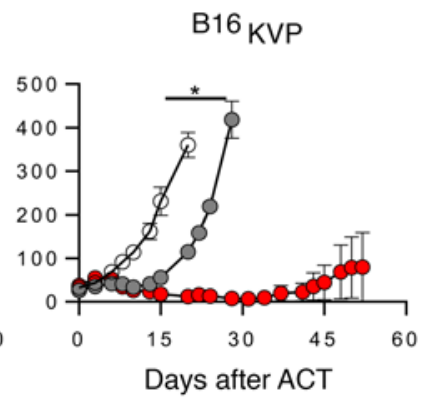

${ }^{\mathrm{B} 16} \mathrm{KVP}^{/ \mathrm{D}^{\mathrm{b}}}$

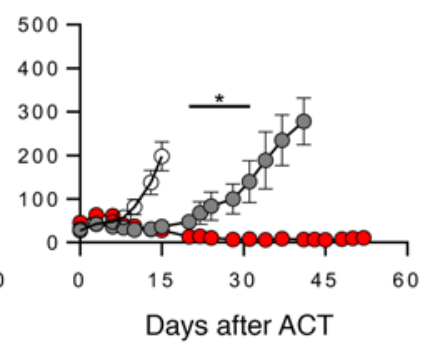

Pmel-1 + vaccine
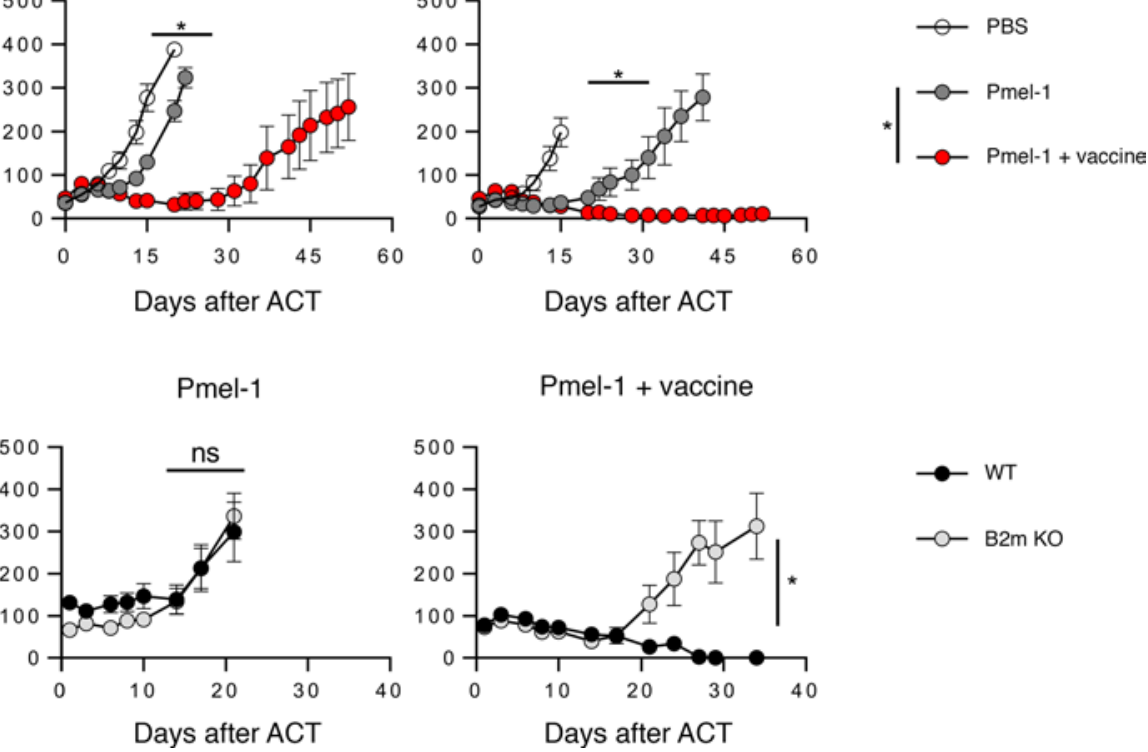

Figure 2. Treatment of modified B16 tumor with adoptively transferred pmel-1 T cells. (A) Tumor treatment scheme. (B) Post-ACT tumor growth curve. Open circles represent mice receiving only irradiation and rhIL-2. Gray circles represent mice treated with $1 \times 10^{6}$ pmel- 1 T cells in addition to radiation and rhlL-2. Red circles represent mice treated with $1 \times 10^{6}$ pmel-1 T cells and rVVhgp100 vaccine in addition to irradiation and rhIL-2. Four to five mice were included in each group. The results represent 1 of 3 independent experiments. Error bars indicate the mean \pm SEM. ${ }^{*} P<0.05$ and $\mathrm{NS}$ indicates no significant differences by Wilcoxon rank-sum test in comparison of tumor growth curve slopes between correspondent groups. (C) Effects of antigen cross-presentation. Tumor injection and irradiation were done as outlined in the scheme in $\mathbf{A}$. Tumor-bearing C57BL/6 mice (black circles) or $\beta 2 \mathrm{mKO}$ mice (gray circles) were treated with a regimen of either rhIL-2 alone, rhIL-2 and $1 \times 10^{6}$ pmel-1 T cells, or rhIL-2, $1 \times 10^{6}$ pmel- 1 T cells, and rVVhgp100 vaccination. Four to five mice were included in each group. The results represent 1 experiment. Error bars indicate the mean \pm SEM. ${ }^{*} P<0.05$ and NS indicates no significant differences by Wilcoxon rank-sum test in comparison of tumor growth curve slopes between WT and $\beta 2 \mathrm{mKO}$ mice.

Compared with endogenous CD25 expression, approximately 50\% of pmel-1 T cells retrovirally transduced with CD25 increased their mean fluorescence intensities (MFIs) after CD25 antibody staining (Figure 5A). When these CD25-transduced and mock-transduced control pmel-1 cells were adoptively transferred into tumor-bearing mice, we did not observe any significant therapeutic effect 
A
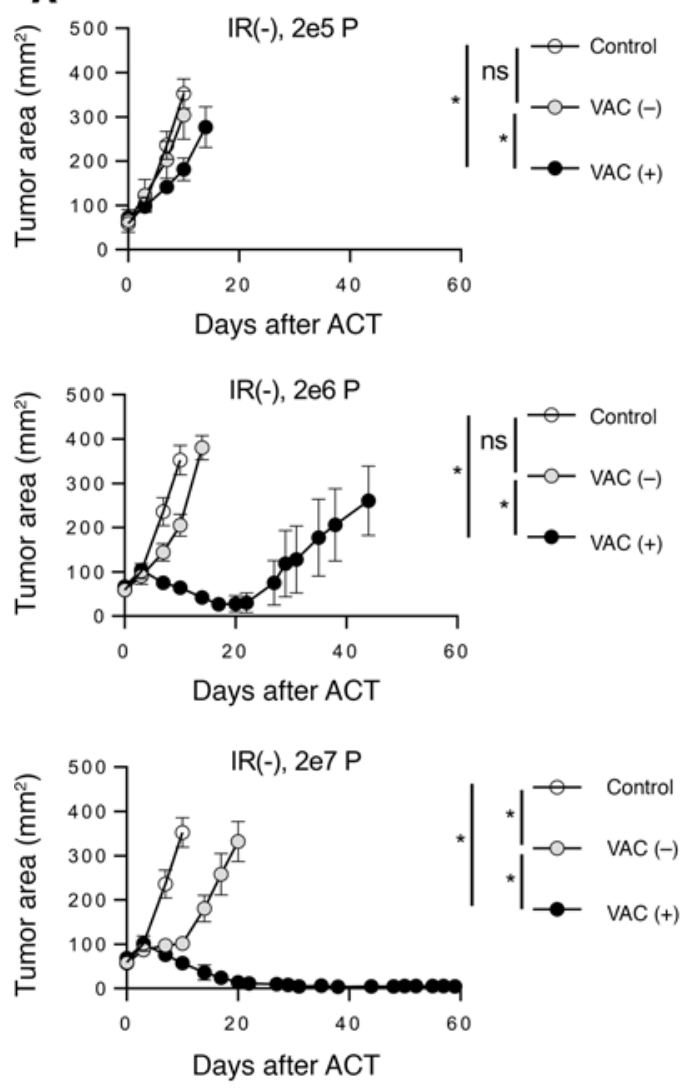

\section{B}

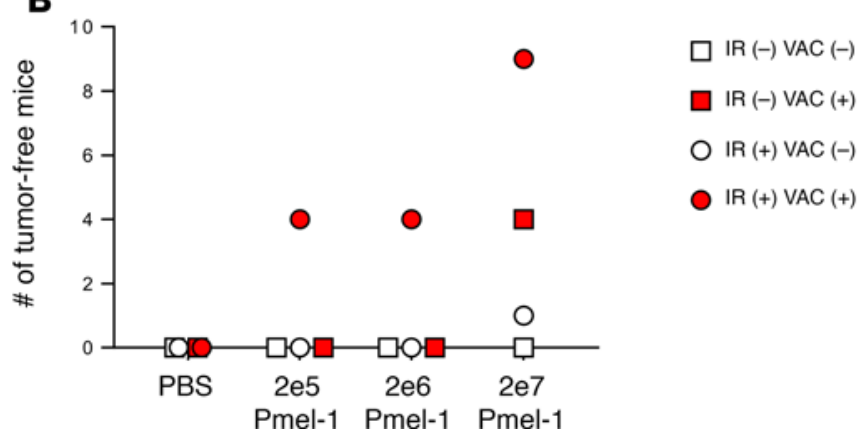

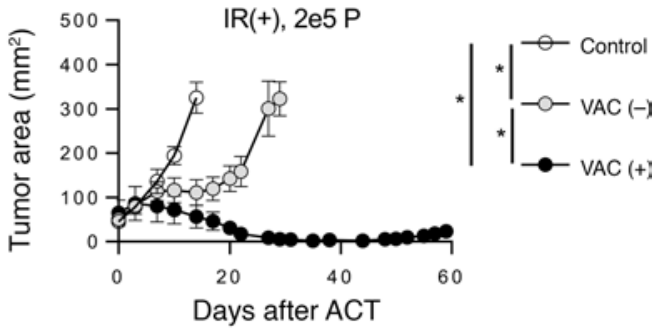
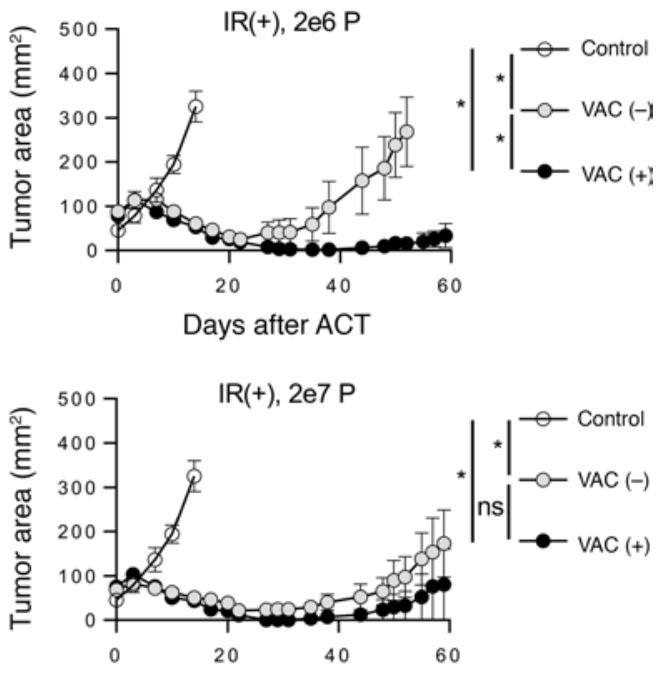

Days after ACT

Figure 3. Effects of pmel-1 T cell numbers in neoantigen-targeting ACT model. (A) C57BL/6 mice were inoculated with $5 \times 10^{5}$ B16 $/ D^{\mathrm{b}}$ tumor cells 11 days before treatment. One day before ACT, mice were irradiated with O [IR(-)] or 5 Gy $[\mathrm{IR}(+)]$. On the day of ACT, mice were injected i.v. With PBS (control, open circles), pmel-1 T cells [VAC (-), gray circles] at indicated doses, or pmel-1 T cells mixed with $2 \times 10^{7}$ PFU of rVVhgp100 [VAC (+), black circles]. A daily dose of 180,000 IU of rhIL-2 was administrated i.p. into all mice for 3 days after ACT. There were 5 mice in each group. Error bars indicate the mean \pm SEM. ${ }^{*} P<$ 0.05 and NS indicates no significant differences by Wilcoxon rank-sum test in comparison of tumor growth curve slopes between correspondent groups. Results represent 1 of 2 independent experiments. (B) Tumor-free mice in combined 2 experiments. Open squares represent without irradiation or vaccination group; red squares represent without irradiation with vaccination group; open circles represent with irradiation without vaccination group; red circles represent with irradiation and vaccination group. Ten mice were included in each group.

of CD25-overexpressing pmel-1 cells targeting the parental-B16- or B16 ${ }_{\mathrm{EGS}}$-injected mice. However, in the case of $\mathrm{B} 16_{\mathrm{KVP}}$-injected mice, pmel-1/CD25 cells regressed the tumor completely with no detectable tumor after 60 days (Figure 5B). Our findings suggest that overexpression CD25 on transferred $\mathrm{T}$ cells may give them advantages in competing for IL-2 with regulatory $\mathrm{T}$ cells, which also express a higher level of CD25 (24), and may in turn allow for less clinical administration of IL-2 during ACT treatment. This experiment showed the sensitive nature of the neoantigen-targeted model to identify a beneficial $\mathrm{T}$ cell modification that was not discernible in the original autoantigen model. 
A

$\operatorname{IR}(-)$

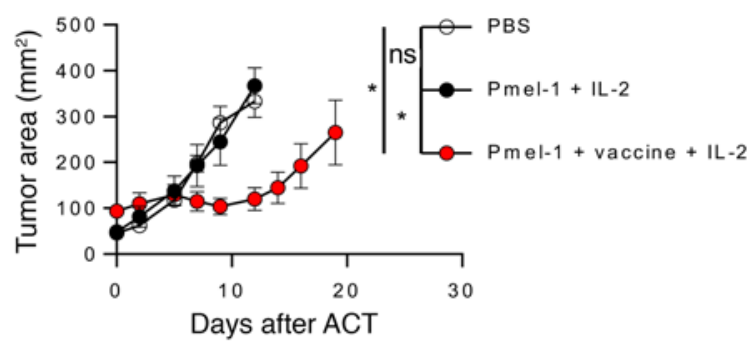

B

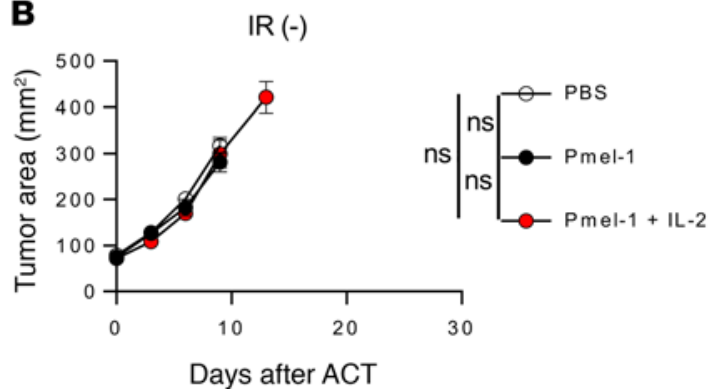

$\operatorname{IR}(+)$

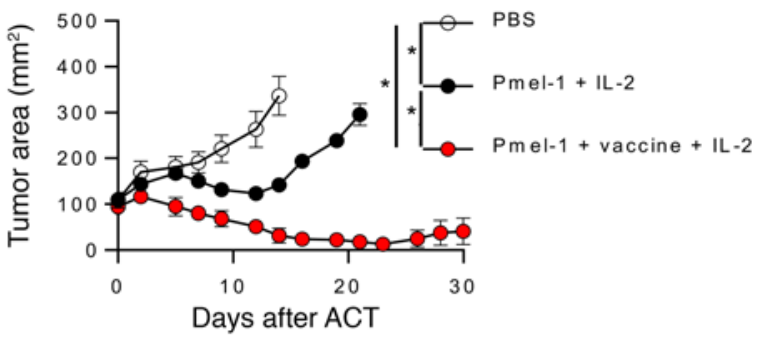

$\operatorname{IR}(+)$

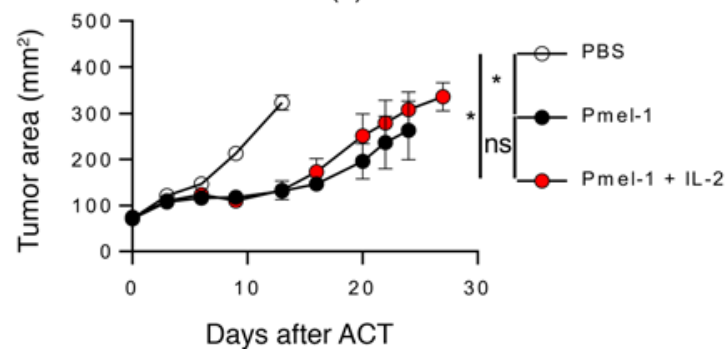

Figure 4. Determinants of neoantigen-targeting ACT model. (A) Effects of sublethal total-body irradiation and vaccine in treating B16 ${ }_{\mathrm{KVP}}$ tumor.

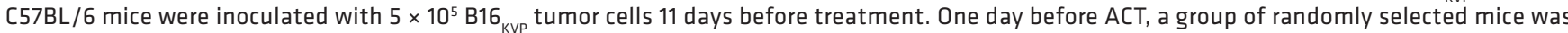
irradiated with 5 Gy. On the day of ACT, mice were injected i.v. with PBS (open circles), $1 \times 10^{6}$ pmel-1 T cells (black circles), or $1 \times 10^{6}$ pmel- 1 T cells mixed with $2 \times 10^{7}$ PFU of rVVhgp100 (red circles). Three daily doses of 180,000 IU of rhIL-2 was administrated i.p. into all mice. There were 5 mice in each group. Error bars indicate the mean \pm SEM. ${ }^{*} P<0.05$ by Wilcoxon rank-sum test in comparison of tumor growth curve slopes between PBSand pmel-1-treated groups. NS, no significant differences by Wilcoxon rank-sum test in comparison of tumor growth curve slopes between pmel-1 and pmel-1 plus vaccination groups. Results represent 1 of 2 independent experiments. (B) Effects of sublethal total-body irradiation and rhIL-2. Tumor injection and irradiation were the same as in A. On the day of ACT, mice were injected i.v. with PBS alone (open circles), $1 \times 10^{6} \mathrm{pmel}-1 \mathrm{~T}$ cells alone (black circles), or pmel-1 T cells plus 3 daily doses of 180,000 rhIL-2 i.p. (red circles). Five mice were included in each group. The results represent 1 of 2 independent experiments. Error bars indicate the mean \pm SEM. NS, no significant differences by Wilcoxon rank-sum test in comparison of tumor growth curve slopes between pmel-1 and pmel-1 plus IL-2 groups. ${ }^{*} P<0.05$ by Wilcoxon rank-sum test in comparison of tumor growth curve slopes between PBS- and Pmel-1-treated groups.

\section{Discussion}

In this study, we created a panel of B16 melanoma derivatives expressing a "mutated self-antigen" targeted by autoreactive pmel-1 T cells and examined the effect of a neoantigen in an ACT treatment model. Neoantigen expression in our model significantly improved tumor immunogenicity and recognition by pmel-1 $\mathrm{T}$ cells. This neoantigen-expressing B16 tumor served as a viable target to model the ACT therapies used in clinic.

Lymphodepletion can be a cause of complications, especially in older patients or in patients who have otherwise experienced chemotherapy or radiation before immunotherapy (25). This can result in a delay in recovery of myeloid cells, lymphocytes, and platelets. Bone marrow toxicity also imposes a restriction on the treatment schedule by making the split dosing or retreatment difficult. In this neoantigen model, we demonstrated complete tumor regression by ACT therapy in combination with vaccine in the absence of lymphodepletion, which was not achievable using the autoantigen model. Hence, there is an opportunity using a neoantigen-targeted model to develop a non-lymphodepleting ACT treatment for patients with epithelial cancers and bone marrow fragility.

Although vaccines based on neoantigens may be one answer for the non-lymphodepleting ACT treatment, translating our observations from mouse to human is rarely straightforward. First, unlike common vaccines used to induce a de novo $\mathrm{T}$ cell response, a vaccine for ACT therapy must be able to stimulate many $\mathrm{T}$ cells at once. Very large numbers of $\mathrm{T}$ cells, in the range of $10^{10}$ to $10^{11}$ cells, are often used in the treatment of solid tumors. Second, our model involves a single, immunogenic neoantigen that is highly expressed. However, in reality, the efficacy of immunizations in activating the breadth and absolute number of cells required to regress a tumor must be questioned when the targeted tumor cell line is heterogeneous with a plethora of mutations expressed at 
A

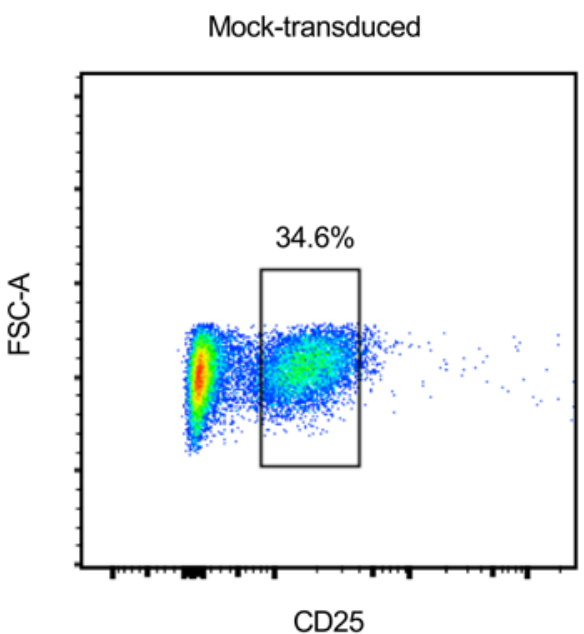

B

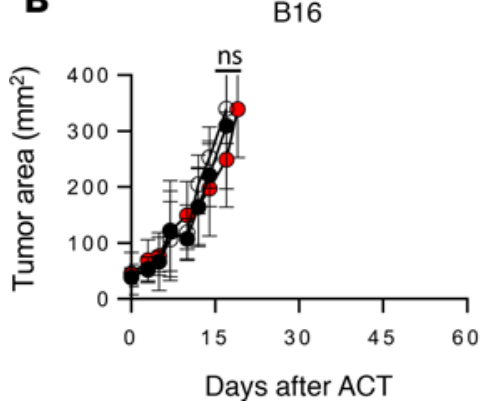

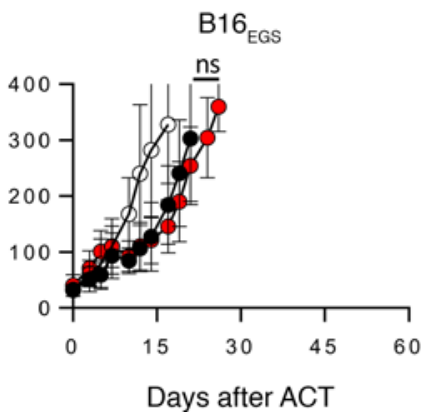

$B 16_{\mathrm{EGS}}$

Days after ACT

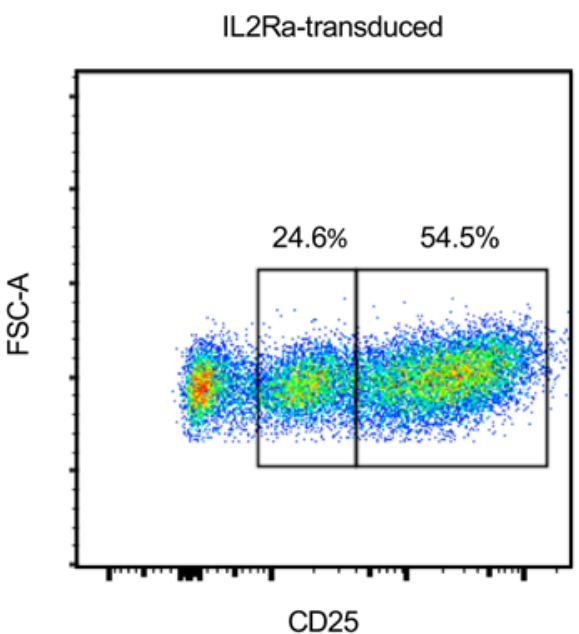

$B 16_{\text {KVP }}$

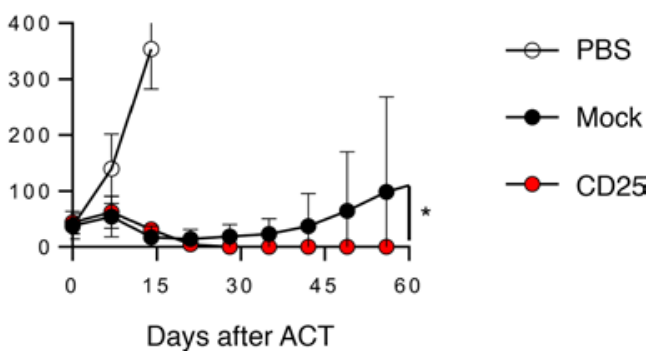

Figure 5. Application of neoantigen model to discover enhanced antitumor functions of ACT. (A) FACS plots of pmel-1 cells transduced with MSGV1-IL$2 \mathrm{Ra}$ or mock retrovirus. (B) Post-ACT tumor growth curve. C57BL/6 mice were injected with $5 \times 10$ B16, B16 ${ }_{\mathrm{ECS}}$, or B16 ${ }_{\mathrm{KVP}}$ subcutaneously. Five Grays irradiation was applied to the mice on day 10, and ACT was given on day 11. All mice were given 180,000 IU of rhIL-2 after ACT for 3 days. In each tumor setting, 5 mice were included in groups receiving PBS (open circles), $1 \times 10^{6}$ mock-transduced pmel- 1 cells (black circles), or $1 \times 10^{6}$ CD25-transduced pmel- 1 cells (red circles). Error bars indicate the mean \pm SEM. ${ }^{*} P<0.05$ by Wilcoxon rank-sum test in comparison of tumor growth curve slopes between groups transferring with CD25- and mock-transduced pmel-1 T cells. NS, no significant differences. The results represent 1 of 2 independent experiments.

varying degrees $(1,26)$. The effectiveness of tumor-specific vaccines in humans is still incompletely elucidated, and most of our understanding regarding their effectiveness is in mouse models with short intervals between tumor introduction and vaccination. We showed that pmel-1 cells recognize the $\mathrm{B} 16_{\mathrm{KVP}}$ tumor-expressed neoantigen without vaccination and further demonstrated their therapeutic effect in transient tumor regression.

Finally, we reexamined the therapeutic effect of IL-2 in ACT. Recently, Sockolosky et al. (27) attempted to solve the toxicity problem in an autoantigen-targeted B16/pmel-1 model by transducing $\mathrm{T}$ cells with modified IL-2 receptors and giving recombinant IL-2. Their modification successfully ameliorated the toxic effect of WT IL-2 but did not result in the better tumor response. We investigated whether increased IL-2 receptor expression on pmel-1 cells bettered the therapeutic response in targeting neoantigen-expressing tumors. We found that overexpression of CD25 in pmel-1 cells significantly improved the therapeutic effect in the neoantigen. In the past decade, many $\mathrm{T}$ cell modification methods were identified using the B16/pmel-1 model (28-31). As our new model using $\mathrm{B} 16_{\mathrm{KVP}}$ or $\mathrm{B} 16_{\mathrm{KVP}} / \mathrm{D}^{\mathrm{b}}$ better mimics $\mathrm{ACT}$ therapy in human malignancies, it will be useful for identifying truly favorable $\mathrm{T}$ cell properties (32-35). We plan to use the $\mathrm{B} 16_{\mathrm{KVP}}$ neoantigen model to further understand $\mathrm{T}$ cell modifications that reduce toxicities and improve the efficacy of $\mathrm{T}$ cell-based cancer immunotherapies.

\section{Methods}

Mice. Four- to six-week-old female C57BL/6NCR mice were purchased from Charles River Laboratories. Pmel-1 TCR-transgenic mice were developed and maintained in our laboratory (19). Four- to six-week-old female $\beta_{2}$-microglobulin-knockout mice (B6.129P2-B2 $m^{\text {tm } I \text { Unc }} / \mathrm{J}$ ) were purchased from The Jackson Laboratory. All mouse experiments were approved under institutional animal study protocol by the Animal Care and Use Committee of the NCI. 
Cell culture. The B16 melanoma line (ATCC) was cultured in complete medium (CM): RPMI 1640 with 10\% FBS, $1 \mathrm{mM}$ sodium pyruvate (Thermo Fisher Scientific), $1 \times$ Non-Essential Amino Acids (Thermo Fisher Scientific), $55 \mu \mathrm{M}$ 2-mercaptoethanol (Gibco), 1× antibiotic-antimycotic (Thermo Fisher Scientific), and $50 \mu \mathrm{g} / \mathrm{ml}$ gentamicin (Gibco). Modified B16 cell lines were maintained in CM with blasticidin S $(10 \mu \mathrm{g} / \mathrm{ml}$; Invivogen) or puromycin ( $5 \mu \mathrm{g} / \mathrm{ml}$; Invivogen). Pmel-1 and CD25-transduced pmel-1 T cells were cultured in CM with $30 \mathrm{IU} / \mathrm{ml}$ recombinant human IL-2 (rhIL-2; Prometheus Laboratories). IFN- $\gamma$ treatment was done by addition of $50 \mathrm{ng} / \mathrm{ml}$ recombinant mouse IFN- $\gamma$ (PeproTech) for 2 days before the detection of $\mathrm{H}-2 \mathrm{D}^{\mathrm{b}}$ by flow cytometry.

Antibodies for flow cytometry, ELISA, and Western blot. HRP-conjugated anti-V5 antibody (R961-25, Thermo Fisher Scientific) and THE beta Actin Antibody [HRP] (2D1D10, Genscript) were used for the detection of the V5 tag and actin, beta by Western blot. For flow cytometry, anti-H-2D ${ }^{\mathrm{b}}$-FITC (KH95, BD Biosciences), anti-mouse CD25-PE (PC61, BD Biosciences), anti-mouse V $\beta 13$ TCRFITC (MR12-3, BD 35 Biosciences), anti-mouse CD8-PE/Cy7 (53-6.7, BioLegend), and anti-mouse CD38-APC/Cy7 (145-2C11, BioLegend) were used.

Production of retroviral constructs. cDNA of Pmel was PCR-amplified from B16 melanoma cells and subcloned into the gamma retroviral vector MSGV1 with the internal ribosome entry site (IRES) and blasticidin resistance gene. Site-directed mutagenesis to change the encoded amino acid sequences from EGS to KVP was performed by the Quick-Change protocol (Agilent) following the manufacturer's protocol. cDNA of Il2ra (CD25) and H2-D1 (H-2D b) was chemically synthesized (IDT DNA) and subcloned into the MSGV1 vector (36) or the MSGV1 vector with IRES and puromycin resistance gene.

Retroviral transduction. For the transduction of B16 cells, retrovirus was generated by transiently transfecting 293gp cells (gift of Paul Robbins, NCI, Bethesda, Maryland, USA) with MSGV1 vectors and the VSVG envelope-encoding plasmid pMD.G. Culture supernatants including retroviral particles and Polybrene ( $8 \mu \mathrm{g} / \mathrm{ml}$; Sigma-Aldrich) were filtered through $0.45-\mu \mathrm{m}$ syringe filters and were added to B16 cells plated the day before. After 8 hours, Polybrene was diluted by addition of the same volume of CM. Cells were split avoiding overgrowth, and 2 days after the transduction, pertinent selection antibiotics were added. For the transduction of pmel-1 T cells, retrovirus was produced by transiently transfecting Platinum Eco cells (Cell Biolabs) with pCL-Eco (Addgene plasmid 12371) and pMSGV1 CD25 or empty pMSGV1 for the mock transduction. Virus particles were loaded onto plate-coated Retronectin (Takara Bio) following the manufacturer's protocol. Pmel- 1 splenocytes were stimulated by addition of $100 \mathrm{ng} / \mathrm{ml}$ each of purified NA/LE hamster anti-mouse CD3 $\varepsilon$ (2C11, BD Pharmingen) and CD28 (37.51, BD Pharmingen) antibodies in CM with $30 \mathrm{IU} / \mathrm{ml}$ of rhIL-2. On the next day of the stimulation, pmel-1 cells were infected by spinoculation at $1000 \mathrm{~g}$ for 10 minutes in virus-loaded plates.

Tumor treatment. Mice were subcutaneously injected with $5 \times 10^{5}$ tumor cells. Ten days later, tumor-bearing mice received $5 \mathrm{~Gy}$ of total-body irradiation. On day 11 after tumor inoculation, mice were treated with cultured pmel-1 T cells i.v. with or without $2 \times 10^{7} \mathrm{PFU}$ of recombinant human gp100-vaccinia (rVVhgp100). In addition, 180,000 IU of rhIL-2 was injected i.p. into mice daily for 3 days after cell transfer. Tumor treatment and measurement were conducted by independent investigators in a double-blinded manner.

Statistics. The slope of the tumor growth measurements was calculated with the Excel (Microsoft) SLOPE function, and the slopes for 2 groups were compared using tumor growth analysis (TGA) software developed in house based on Wilcoxon rank-sum test. A $P$ value less than 0.05 was considered significant. IFN- $\gamma$ concentration in pmel-1 and tumor cell line coculture assays was compared and analyzed by ANOVA followed by Tukey's multiple-comparisons test. A $P$ value less than 0.01 was considered significant. Prism 8.0 (GraphPad) was used for the analysis.

Study approval. All mouse experiments were approved under institutional animal study protocol by the Animal Care and Use Committee of the National Cancer Institute, Bethesda, Maryland, USA.

\section{Author contributions}

$\mathrm{KH}$ conceived the model, established cell lines, designed experiments, conducted experiments, and wrote the manuscript. ZY designed research studies, conducted experiments, acquired and analyzed data, provided reagents, and wrote the manuscript. GRC analyzed data and wrote the manuscript. ASP conducted experiments and edited the manuscript. NPR interpreted data, wrote and edited the manuscript, and has principal responsibility for this report. 


\section{Acknowledgments}

The authors thank David W. Jones for his technical support in animal experiments and Donald White for developing tumor growth analysis software. The research was funded by the Intramural Research Program, by the Cancer Moonshot program for the Center for Cell-Based Therapy at the NCI, NIH, and by the Milstein Family Foundation. The authors also thank James C. Yang and S.A. Rosenberg for their valuable discussions and intellectual input.

Address correspondence to: Zhiya Yu or Nicholas P. Restifo, 9000 Rockville Pike, Building 10CRC, Room 3w-5816, Bethesda, Maryland 20892, USA. Phone: 240.858.3812; Email: zhiya_yu@nih.gov (ZY). Phone: 240.760.6217; Email: Restifo@nih.gov (NPR).

1. Robbins PF, et al. A pilot trial using lymphocytes genetically engineered with an NY-ESO-1-reactive T-cell receptor: long-term follow-up and correlates with response. Clin Cancer Res. 2015;21(5):1019-1027.

2. Goff SL, et al. Enhanced receptor expression and in vitro effector function of a murine-human hybrid MART-1-reactive T cell receptor following a rapid expansion. Cancer Immunol Immunother. 2010;59(10):1551-1560.

3. Pardoll D. Does the immune system see tumors as foreign or self? Annu Rev Immunol. 2003;21:807-839.

4. Hogquist KA, Baldwin TA, Jameson SC. Central tolerance: learning self-control in the thymus. Nat Rev Immunol. 2005;5(10):772-782

5. Rosenberg SA, Restifo NP. Adoptive cell transfer as personalized immunotherapy for human cancer. Science. 2015;348(6230):62-68.

6. Greenman C, et al. Patterns of somatic mutation in human cancer genomes. Nature. 2007;446(7132):153-158.

7. Vogelstein B, Papadopoulos N, Velculescu VE, Zhou S, Diaz LA, Kinzler KW. Cancer genome landscapes. Science. 2013;339(6127):1546-1558.

8. Lawrence MS, et al. Mutational heterogeneity in cancer and the search for new cancer-associated genes. Nature. 2013;499(7457):214-218.

9. Tran E, et al. Cancer immunotherapy based on mutation-specific CD4 ${ }^{+} \mathrm{T}$ cells in a patient with epithelial cancer. Science. 2014;344(6184):641-645.

10. Tran E, et al. T-cell transfer therapy targeting mutant KRAS in cancer. N Engl J Med. 2016;375(23):2255-2262.

11. Zacharakis $\mathrm{N}$, et al. Immune recognition of somatic mutations leading to complete durable regression in metastatic breast cancer. Nat Med. 2018;24(6):724-730.

12. Tran E, Robbins PF, Rosenberg SA. 'Final common pathway' of human cancer immunotherapy: targeting random somatic mutations. Nat Immunol. 2017;18(3):255-262.

13. Hanson HL, et al. Eradication of established tumors by CD8 ${ }^{+} \mathrm{T}$ cell adoptive immunotherapy. Immunity. 2000;13(2):265-276

14. Castle JC, et al. Exploiting the mutanome for tumor vaccination. Cancer Res. 2012;72(5):1081-1091.

15. Matsushita $\mathrm{H}$, et al. Cancer exome analysis reveals a T-cell-dependent mechanism of cancer immunoediting. Nature. 2012;482(7385):400-404.

16. Fehlings $\mathrm{M}$, et al. Checkpoint blockade immunotherapy reshapes the high-dimensional phenotypic heterogeneity of murine intratumoural neoantigen-specific CD8. Nat Commun. 2017;8(1):562.

17. Dankort D, et al. Braf(V600E) cooperates with Pten loss to induce metastatic melanoma. Nat Genet. 2009;41(5):544-552.

18. Acquavella N, et al. Type I cytokines synergize with oncogene inhibition to induce tumor growth arrest. Cancer Immunol Res. 2015;3(1):37-47.

19. Overwijk WW, et al. Tumor regression and autoimmunity after reversal of a functionally tolerant state of self-reactive CD8 ${ }^{+} \mathrm{T}$ cells. J Exp Med. 2003;198(4):569-580.

20. Overwijk WW, et al. gp100/pmel 17 is a murine tumor rejection antigen: induction of "self"-reactive, tumoricidal T cells using high-affinity, altered peptide ligand. J Exp Med. 1998;188(2):277-286.

21. Robbins PF, et al. Mining exomic sequencing data to identify mutated antigens recognized by adoptively transferred tumor-reactive T cells. Nat Med. 2013;19(6):747-752.

22. Wrzesinski C, et al. Increased intensity lymphodepletion enhances tumor treatment efficacy of adoptively transferred tumor-specific T cells. J Immunother. 2010;33(1):1-7.

23. Su EW, et al. IL-2R $\alpha$ mediates temporal regulation of IL-2 signaling and enhances immunotherapy. Sci Transl Med. 2015;7(311):311ra170.

24. Sakaguchi S, Sakaguchi N, Asano M, Itoh M, Toda M. Immunologic self-tolerance maintained by activated T cells expressing IL-2 receptor $\alpha$-chains (CD25). Breakdown of a single mechanism of self-tolerance causes various autoimmune diseases. $J$ Immunol. 1995;155(3):1151-1164.

25. Muranski P, et al. Increased intensity lymphodepletion and adoptive immunotherapy — how far can we go? Nat Clin Pract Oncol. 2006;3(12):668-681.

26. Klebanoff CA, Yu Z, Hwang LN, Palmer DC, Gattinoni L, Restifo NP. Programming tumor-reactive effector memory CD8 ${ }^{+} \mathrm{T}$ cells in vitro obviates the requirement for in vivo vaccination. Blood. 2009;114(9):1776-1783.

27. Sockolosky JT, et al. Selective targeting of engineered T cells using orthogonal IL-2 cytokine-receptor complexes. Science. 2018;359(6379):1037-1042.

28. Palmer DC, et al. Cish actively silences TCR signaling in CD8+ T cells to maintain tumor tolerance. J Exp Med. 2015;212(12):2095-2113.

29. Ji Y, et al. miR-155 augments $\mathrm{CD} 8^{+} \mathrm{T}$-cell antitumor activity in lymphoreplete hosts by enhancing responsiveness to homeostatic $\gamma$ c cytokines. Proc Natl Acad Sci U S A. 2015;112(2):476-481.

30. Sukumar M, et al. Mitochondrial membrane potential identifies cells with enhanced stemness for cellular therapy. Cell Metab. 
2016;23(1):63-76.

31. Clever D, et al. Oxygen sensing by T cells establishes an immunologically tolerant metastatic niche. Cell. 2016;166(5):1117-1131.e14.

32. Eil R, et al. Ionic immune suppression within the tumour microenvironment limits $\mathrm{T}$ cell effector function. Nature. 2016;537(7621):539-543.

33. Yamamoto TN, et al. T cells genetically engineered to overcome death signaling enhance adoptive cancer immunotherapy. J Clin Invest. 2019;129(4):1551-1565.

34. Patel SJ, et al. Identification of essential genes for cancer immunotherapy. Nature. 2017;548(7669):537-542.

35. Vodnala SK, et al. T cell stemness and dysfunction in tumors are triggered by a common mechanism. Science. 2019;363(6434):eaau0135.

36. Morgan RA, et al. Cancer regression in patients after transfer of genetically engineered lymphocytes. Science. 2006;314(5796):126-129. 